\title{
The Italian Network for Tumor Biotherapy (NIBIT): past, present and future goals
}

Michele Maio ${ }^{1,2}$, Ester Fonsatti ${ }^{1,2}$

1 Medical Oncology and Immunotherapy, Department of Oncology, University Hospital of Siena, Istituto Toscano Tumori, Siena, Italy

2 NIBIT - Network Italiano per la Bioterapia dei Tumori

Immunotherapy and target therapy are providing compelling proofs of clinical success in malignant melanoma, strengthening their role as additional options in the comprehensive treatment of melanoma patients. In particular, immunomodulating monoclonal antibodies $(\mathrm{mAb})$ that block specific immune checkpoints and BRAF kinase inhibitors have been demonstrated to improve significantly the overall survival (OS) of metastatic melanoma patients [1,2]. More interestingly, results from pre-clinical studies and ongoing clinical trials suggest that these agents can also have a strong clinical impact in additional human malignancies beyond melanoma [3], either in combination with each other, or with immunotherapeutic or chemotherapeutic agents [4]. However, the swift clinical translation of emerging evidences on new therapeutic strategies and agents from the laboratory to the clinical setting is generally long-winded, raising new and specific procedural, ethical, and legal challenges that cannot be easily faced by single Institutions. Thus, to facilitate the clinical application of promising pre-clinical evidences generated by non-profit entities, shared efforts and interactions among different professionals are mandatory. To pursue this common goal, different national networks focusing on immunobiotherapy of cancer are being established in Europe. In this broad context, to fulfill the needs of the Italian scientific community, the NIBIT (acronym for the Network Italiano per la Bioterapia dei Tumori - Italian Network for Tumor Biotherapy) was established in 2004, and was formally given legal status as a non-profit association in 2006. As of today, the NIBIT accounts for more than 150 official members from over 40 Italian Academic, Regulatory and Industrial groups/bodies nationwide.

As per its Statute, the activities of the NIBIT (available at http://www.nibit.org; temporarily at http:// www.sienaweb.it/nibit) aim to:

- Promote and foster scientific and operative interactions among professionals belonging to Academia, Biotech/Pharmaceutical Industry, Regulatory Bodies;

- Set-up initiatives to inform patients on cancer bioimmunotherapy and available ongoing clinical trials; 


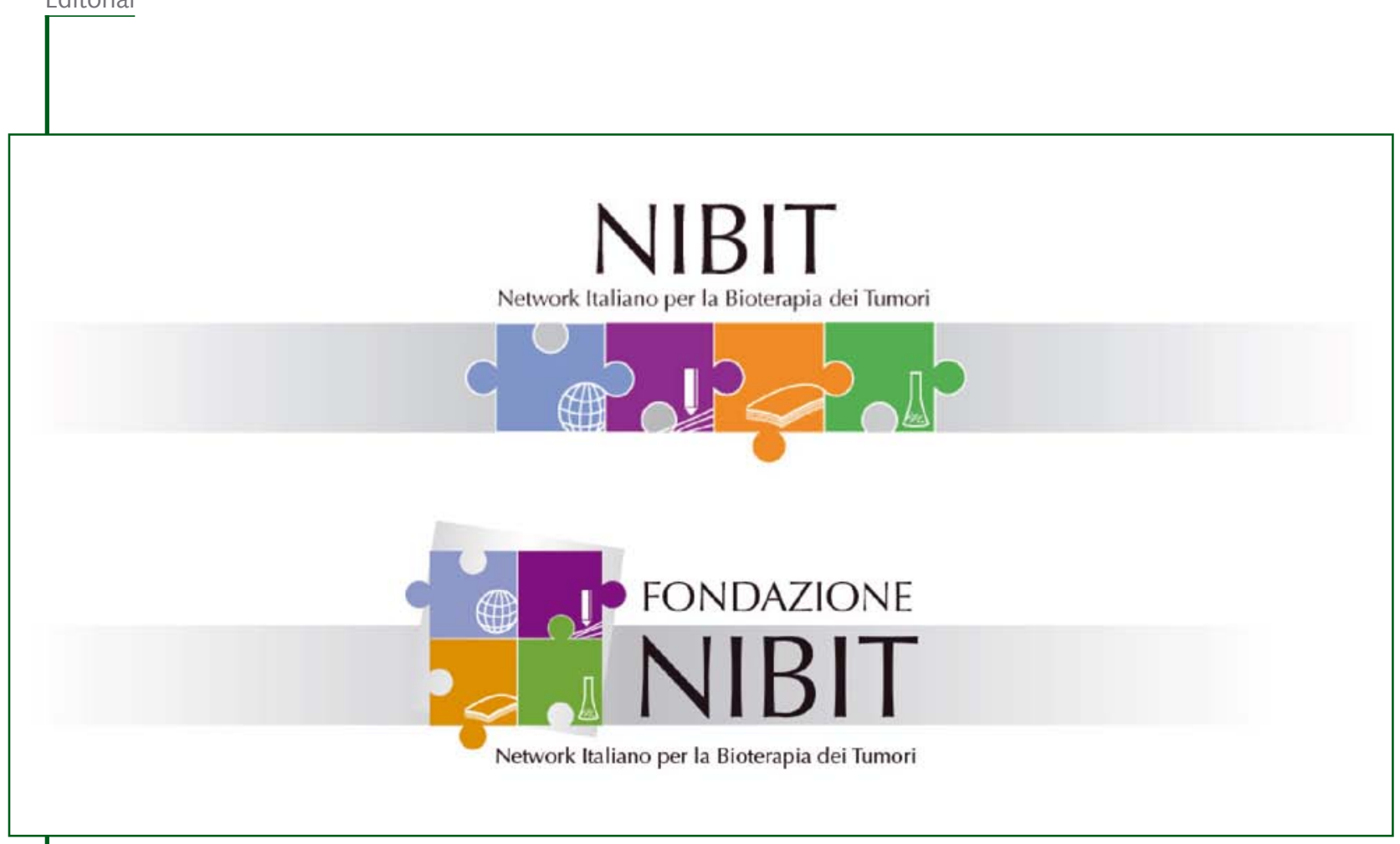

Figure 1. NIBIT (upper) and NIBIT Foundation (below) logo

- Train physicians about potentials and limitations of cancer bio-immunotherapy, and on available clinical trials;

- Develop innovative, multi-center clinical studies of cancer bio-immunotherapy at national level.

These objectives are well identified by the NIBIT logo: 4 intermingled pieces of a puzzle (interaction, training, information and research) of different colors (Figure 1).

The NIBIT held its scientific annual meeting at the Certosa di Pontignano, a Tuscan monastery built by the Chartusian Order in 1343 that is located in the beautiful Chianti's landscape. The annual meeting allows to present the most recent pre-clinical results obtained by the NIBIT groups as well as the clinical activities of the Network in the context of a high level international framework. A scientific report is generated after the meeting and it is hosted by Cancer Immunology Immunotherapy, a peer reviewed international journal focused on basic, translational, and clinical cancer immunology and immunotherapy, that starting from 2013 is the official scientific Journal of the NIBIT. Since 2013 the annual NIBIT meeting is organized within the framework of the international meeting "Cancer Bio-Immunotherapy in Siena" in which world-acknowledged scientists join the Faculty of the NIBIT (http://www. sienameeting-nibit.org). To promote the scientific activities of young researchers, three NIBIT prizes are awarded yearly for the best basic, translational and clinical contributions. To contribute to promote the knowledge on the results and potentials of cancer immunotherapy among the larger clinical and scientific community, as well as to non-dedicated health professionals, members of the NIBIT held four yearly itinerating courses in different Italian locations.

The courses are designed to allow time for extensive discussion between speakers and participants. In 2014, the NIBIT will also organize in Siena the First National Master in Immuno-Oncology, aimed to provide a limited number of attendees with an in depth, practical experience in the clinics of cancer immunotherapy.

In order to better fulfill its mission on a world-wide basis, the NIBIT is member of the World Immunotherapy Council (http://www.sitcancer.org/about-sitc/initiatives/wic), an international forum that groups national organizations to facilitate high-level scientific collaborations in cancer immunology and immunotherapy. 
Most recently the NIBIT has also designed and conducted its first clinical trial entitled: "A Phase II Study of the Combination of Ipilimumab and Fotemustine in Patients with Unresectable Locally Advanced or Metastatic Malignant Melanoma”. This study has involved 8 Italian Institutions with the primary objective to investigate the efficacy of the combination of the anti-CTLA-4 mAb ipilimumab and fotemustine in patients with unresectable locally advanced or metastatic melanoma with or without asymptomatic brain metastases. The initial results of the study have been presented at major international meetings and have been published in The Lancet Oncology in 2012 [4]. The comprehensive success of this clinical study represents a great achievement for the NIBIT and shows the potential of Academic networks to generate high-level scientific research that overcomes the hurdles that new modalities of cancer bio-immunotherapy face.

In spite of the successes that cancer immuno-biotherapy is achieving, much remains to be gained in the identification of the fine immunologic mechanism(s) activated in treated patients, as well as on predictive and prognostic biomarkers that will allow the selection of the best candidates to this therapeutic strategy (Ref 7). The latter represents an area of very active and competitive research; thus, an immune monitoring platform was established and developed by several groups that participated to the NIBITM1 study. To further implement these ex-vivo activities, the first NIBIT laboratory has been established in 2013 to set-up and to perform translational studies foreseen in clinical trials supported or sponsored by the NIBIT.

In the aim to best focus the comprehensive activities connected with the development and conduction of non-profit clinical trials, the NIBIT Board of Directors and its Assembly, have approved the establishment of the NIBIT Foundation (http://www.fondazionenibit.org) in 2012 (Figure 1). Main objectives of the NIBIT Foundation are to:

- Develop, design and conduct clinical Phase I to IV studies;

- Participate in projects aimed at the development of new drugs and new regimens for cancer treatment;

- Coordinate and/or participate in multicenter national and international clinical trials;

- Promote and/or implement scientific initiatives, such as conferences, meetings, seminars, courses, publications;

- Support research projects, including basic and translational programs.

On these premises, the NIBIT Foundation is presently sponsor of the NIBIT-M2 study "A randomized, Phase III study of Fotemustine versus the Combination of Fotemustine and Ipilimumab in Patients with Metastatic Melanoma with brain metastasis" and is working on setting up additional high-level clinical programs. As per the NIBIT, the clinical activities of the NIBIT Foundation aim to further

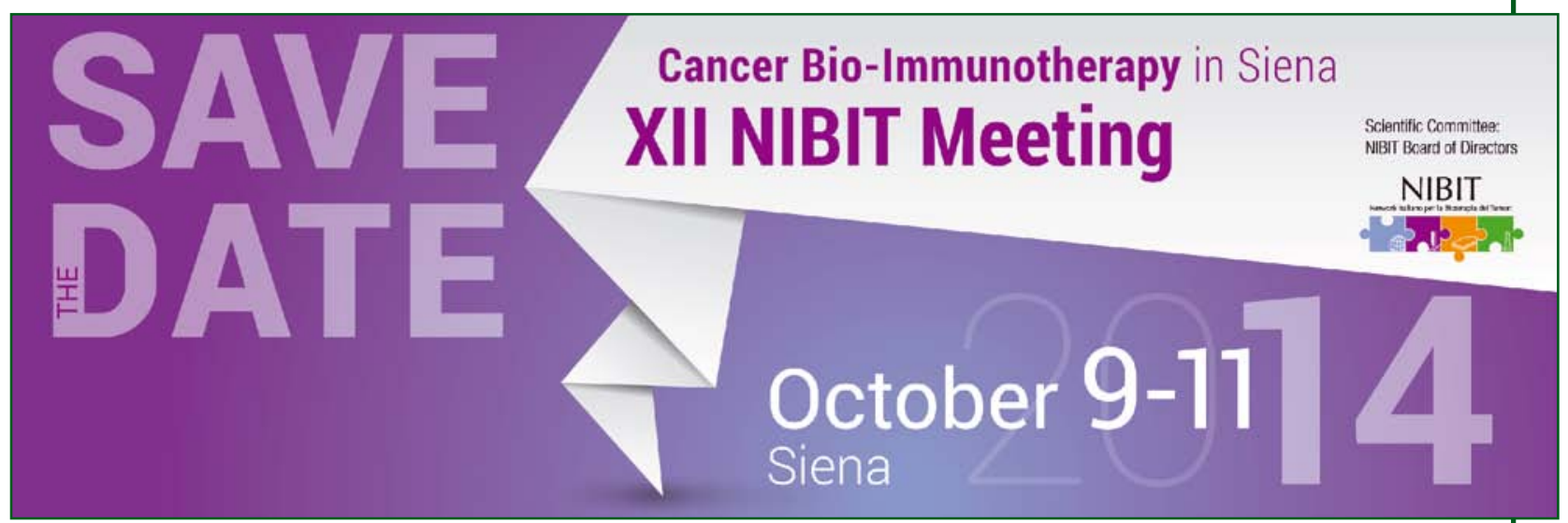

Figure 2. The dates for the next NIBIT meeting 
strengthen operative links within the larger Italian research community, but also with international groups and Institutions focusing on cancer bio-immunotherapy.

Together with the NIBIT Board of Directors, we look forward to seeing you in Siena on October 9-11, 2014 to enjoy the next NIBIT Meeting (Figure 2) and the superb beauty of Siena and of the Tuscan landscapes.

\section{References}

1. Hodi FS, O’Day SJ, McDermott DF, et al. Improved survival with ipilimumab in patients with metastatic melanoma. N Engl J Med 2010; 363: 711-23; http://dx.doi.org/10.1056/NEJMoa1003466

2. Chapman $\mathrm{PB}$, Hauschild $A$, Robert $C$, et al. Improved survival with vemurafenib in melanoma with BRAF V600E mutation. N Engl J Med 2011; 364: 2507-16; http://dx.doi.org/10.1056/NEJMoa1103782

3. Calabrò L, Morra A, Fonsatti E, et al. Tremelimumab for patients with chemotherapy-resistant advanced malignant mesothelioma: an open-label, single-arm, phase 2 trial. Lancet Oncol 2013; 14: 1104-11; http://dx.doi.org/10.1016/S1470-2045(13)70381-4

4. Di Giacomo AM, Ascierto PA, Pilla L, et al. Ipilimumab and fotemustine in patients with advanced melanoma (NIBIT-M1): an open-label, single-arm phase 2 trial. Lancet Oncol 2012; 13: 879-86; http://dx.doi.org/10.1016/S1470-2045(12)70324-8 